\title{
Letters
}

\section{Robust Stability Analysis Using the Small Gain, Circle, Positivity, and Popov Theorems: A Comparative Study}

Wassim M. Haddad, Emmanuel G.

Collins, Jr., and Dennis S. Bernstein

Abstract-This note analyzes the stability robustness of a Maximum Entropy controller designed for a benchmark problem. Four robustness tests are used: small gain analysis, circle analysis, positive real analysis, and Popov analysis, each of which is guaranteed to give a less conservative result than the previous test. The analysis here is performed graphically although recent research has developed equivalent tests based on Lyapunov theory. The Popov test is seen, for this example, to yield highly nonconservative robust stability bounds. The results here illuminate the conservatism of analysis based on traditional small-gain type tests and reveal the effectiveness of analysis tests based on Popov analysis and related parameter-dependent Lyapunov functions.

\section{INTRODUCTION}

In control engineering practice, control design (whether classical or modern) is usually predicated upon some nominal (usually linear) model of the plant to be controlled. However, this nominal model of the system is never an exact representation of the true physical system. This necessitates tools that allow a control system to be analyzed for robustness with respect to errors in the design model. These analysis tools almost always lead to techniques for actually designing a control system for robustness.

In classical control, gain and phase margins are often used as indirect measures of robustness. However, these criteria do not always adequately provide robustness with respect to the true plant uncertainties. Hence, to add reliability to the analysis process, more direct and rigorous measures of robustness are needed. To guarantee the best performance possible, in the presence of uncertainties in the system model, it is important that these robustness measures be nonconservative.

In the analysis of systems for robustness, the conservatism of the resulting robust stability and performance bounds is largely dependent upon the characterization of the uncertainty in the analysis process. This uncertainty characterization can be viewed as lying between two extremes. In the state space, one extreme would be to model the uncertainty as constant, real parameters while the opposite extreme would be to model the uncertainty as arbitrarily time-varying, real parameters. In the frequency domain, the corresponding extremes are to model the uncertainty as a transfer function with bounded phase or oppositely, as a transfer function with arbitrary phase.

If the uncertainty is truly constant and real, then modeling it as arbitrarily time-varying can lead to very conservative results. For example, classical analysis of a Hill's equation (e.g., the Mathieu

Manuscript received February 15, 1993; revised October 11, 1993. This work was supported in part by the National Science Foundation under Grant ECS 9109558 , by the Air Force Office of Scientific Research under Grant F49620-92-J-0127 and Contract F49620-91-C- 0019, and the Florida Space Grant Consortium under Grant NGT.

Wassim M. Haddad is with the Department of Mechanical and Aerospace Engineering, Florida Institute of Technology, Melboume, FL 32901.

Emmanuel G. Collins, Jr. is with the Harris Corporation, Government Aerospace Systems, MS 1914849 Melboume, FL 32902.

IEEE Log number 9214249.

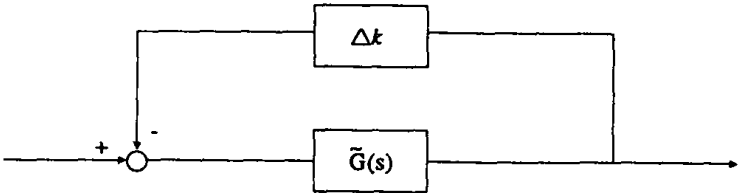

Fig. 1 Standard uncertainty representation.

equation) shows that time-varying parameter variations can destabilize a system even when the parameter variations are confined to a region in which constant variations are nondestabilizing (1). Also, as seen in [2] which analyzes stiffness uncertainty for a flexible structure, when uncertainty is modeled as having arbitrary phase, predictions for stability and performance will be much more conservative than results developed assuming phase-bounded (e.g., positive real) uncertainty.

In recent years it has become conventional to model plant uncertainty, say $\Delta k$, using the feedback configuration shown in Figure 1. In this figure $\tilde{G}(s)$ denotes the nominal plant. Four of the most fundamental results concerning stability of feedback system interconnections are the small gain, circle, positivity, and Popov theorems $[1,3]$. Even though these theorems were originally developed to analyze stability of system with a single, memoryless nonlinear element in a feedback configuration [1], in recent research [3, 4] each result was reinterpreted and generalized to the problem of robust stability involving linear uncertainty. To do this, a Lyapunov function framework was established, providing connections of these classical results to robust stability and performance via state space methods.

As shown in [3], the main difference between the small gain, circle, and positivity theorems versus the Popov theorem is that the former results guarantee robustness with respect to arbitrarily, time-varying uncertainty while the Popov theorem restricts the time variation of the uncertainty. This is not surprising once one recognizes that the Lyapunov function foundation of the small gain, circle, and positivity theorems is based upon conventional or "fixed" quadratic Lyapunov functions which, of course, guarantee stability with respect to arbitrarily, time-varying perturbations. In contrast, the Popov theorem is based upon a quadratic Lyapunov function that is a function of the parametric uncertainty, that is, a parameter-dependent quadratic Lyapunov function [3, 4]. Hence, in effect, the Popov result guarantees stability by means of a family of Lyapunov functions. A key aspect of this approach [4] is the fact that it does not apply to arbitrarily time-varying uncertainties, which renders it significantly less conservative than fixed quadratic Lyapunov functions in the presence of constant real parameter uncertainty.

To illuminate the conservatism of robustness analysis based on traditional small-gain type tests for constant real parameter uncertainty and to reveal the importance of tests which restrict the time-variation in the state space and thus allow the incorporation of phase information in the frequency domain, we consider a simple two-mass/spring, lightly damped, system with uncertain stiffness [5]. This example was chosen to highlight the inherent drawbacks of small gain principles applied to the analysis of feedback systems with constant real parameter uncertainty. A quadratic Lyapunov function framework leading to an algebraic basis in terms of matrix Riccati 


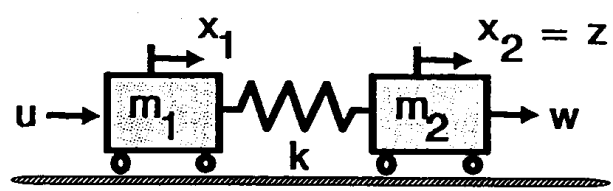

Fig. 2 Spring-mass system.

equations for the analysis and synthesis of robust controllers for the small-gain, circle, positivity, and Popov theorems is given in [3, 4]. Nevertheless, for simplicity the analysis presented here is graphical.

\section{TWO-MASS/SPRING EXAMPLE}

Consider the two-mass/spring system shown in Figure 2 with uncertain stiffness $k$. A control force acts on body 1 , and the position of body 2 is measured resulting in a noncolocated control problem. Here, we consider Controller $\backslash \# 1$ of $[6,7]$ which was designed for Problem $1 \# 1$ of a benchmark problem [5] using the Maximum Entropy robust control design technique. The controller transfer function given by

$$
H(s)=\frac{194390(s+0.33679)\left[(s-0.11735)^{2}+0.90996^{2}\right]}{(s+81.438)(s+131.04)\left[(s+2.9049)^{2}+1.8615^{2}\right]}
$$

was designed so that the closed-loop system is robust with respect to perturbations in the nominal value of the stiffness $k$ (i.e., $k=k_{\mathrm{nom}}$ ). The exact stiffness stability region over which the system will remain stable was computed by a simple search and is given by

$$
0.4459 \leq k \leq 2.0660
$$

Next, using a graphical approach we apply small gain analysis, circle analysis, positive real analysis, and Popov analysis to determine the stiffness stability regions predicted by each of these tests. Each of these tests is related to the previous test and is guaranteed to be less conservative.

We begin by constructing the uncertainty feedback system that will be used in each of the tests. The plant (for $m_{1}=m_{2}=1$ ) is given by the triple $(A(k), B, C)$ where

$$
A(k)=\left[\begin{array}{cccc}
0 & 0 & 1 & 0 \\
0 & 0 & 0 & 1 \\
-k & k & 0 & 0 \\
k & -k & 0 & 0
\end{array}\right], \quad B=\left[\begin{array}{l}
0 \\
0 \\
1 \\
0
\end{array}\right], \quad C=\left[\begin{array}{llll}
0 & 1 & 0 & 0
\end{array}\right]
$$

The perturbation in $A(k)$ due to a change in the stiffness element $k$ from nominal value $k_{\text {nom }}$ is given by

$$
A(k)-A\left(k_{\mathrm{nom}}\right) \triangleq \Delta A=B_{0} \Delta k C_{0}
$$

where $B_{0}^{\mathrm{T}}=\left[\begin{array}{llll}0 & 0 & -1 & 1\end{array}\right]$ and $C_{0}=\left[\begin{array}{llll}1 & -1 & 0 & 0\end{array}\right]$. In the subsequent analysis we will choose $k_{\mathrm{nom}}=0.6$ since the controller (1) was developed under this assumption.

Let the triple $\left(A_{c}, B_{c}, C_{c}\right)$ denote the state space representation of the controller (1). Then, assuming negative feedback, the closed-loop state matrix is given by

$$
\tilde{A}(k)=\left[\begin{array}{cc}
A(k) & -B C_{c} \\
B C_{c} & A_{c}
\end{array}\right]
$$

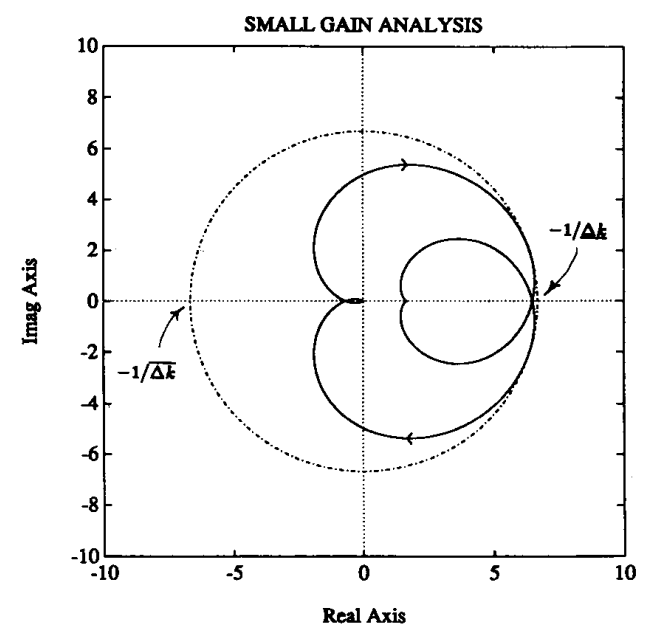

Fig. 3 Small gain analysis.

Next, define $\tilde{B}_{0}^{\mathrm{T}}=\left[\begin{array}{ll}B_{0}^{\mathrm{T}} & 0_{1 \times 4}\end{array}\right], \quad \tilde{C}_{0}=\left[\begin{array}{ll}C_{0} & 0_{1 \times 4}\end{array}\right]$ and let $\tilde{G}(s)=-\tilde{C}_{0}\left(s I-\tilde{A}\left(k_{\text {nom }}\right)\right)^{-1} \tilde{B}_{0}$. Then, the plant uncertainty $\Delta k$ can be represented by a fictitious feedback loop as shown in Figure 1 .

For each of the tests below we will determine $\overline{\Delta k}$ (positive) and $\underline{\Delta k}$ (negative) such that stability is guaranteed for

$$
k_{\mathrm{nom}}+\underline{\Delta k} \leq k \leq k_{\mathrm{nom}}+\overline{\Delta k}
$$

\section{Small Gain Analysis}

Small gain analysis requires considering the Nyquist diagram of $\tilde{G}(s)$. The smallest circle centered at the origin that completely encompasses the Nyquist diagram, $\operatorname{Im}[\tilde{G}(j \omega)]$ vs. $\operatorname{Re}[\tilde{G}(j \omega)]$ for all $\omega$, (without touching it) is then drawn. The intersection of this circle with the negative real axis is given by $-1 / \overline{\Delta k}$ and the intersection with the positive real axis is given by $-1 / \underline{\Delta}$. This analysis is shown in Figure 3. It follows that $\overline{\Delta k}=0.1496$ and $\underline{\Delta k}=-0.1496$. Hence, using small gain analysis, stability is guaranteed for

$$
0.4504 \leq k \leq 0.7496 \text {. }
$$

Note that since the $\Delta k$ uncertainty block is comprised of a single scalar, this result is equivalent to a $\mu$-analysis test [8].

\section{Circle Analysis}

As in small gain analysis, circle analysis determines stability bounds by drawing a circle that completely encompasses the Nyquist diagram (without touching it). However, the circle criterion allows the center of the circle to lie anywhere along the real axis and can hence give a less conservative bound $\overline{\Delta k}$ (or $\underline{\Delta k}$ ) at the expense of increased conservatism in the remaining bound $\underline{\Delta k}$ (or $\overline{\Delta k}$ ). Here we choose the center of the circle to lie at $\left(\left(x_{\min }+x_{\max }\right) / 2,0\right)$ where $x_{\min }$ is the minimum real part of the Nyquist diagram and $x_{\max }$ is the maximum real part. The intersection of this circle with the negative real axis equals $-1 / \overline{\Delta k}$ and the intersection with the positive real axis equals by $-1 / \underline{\Delta k}$. This analysis is shown in Figure 4. It follows that $\overline{\Delta k}=0.3167$ and $\Delta k=-0.1277$. Hence, using circle analysis, stability is guaranteed for

$$
0.4722 \leq k \leq 0.9167
$$




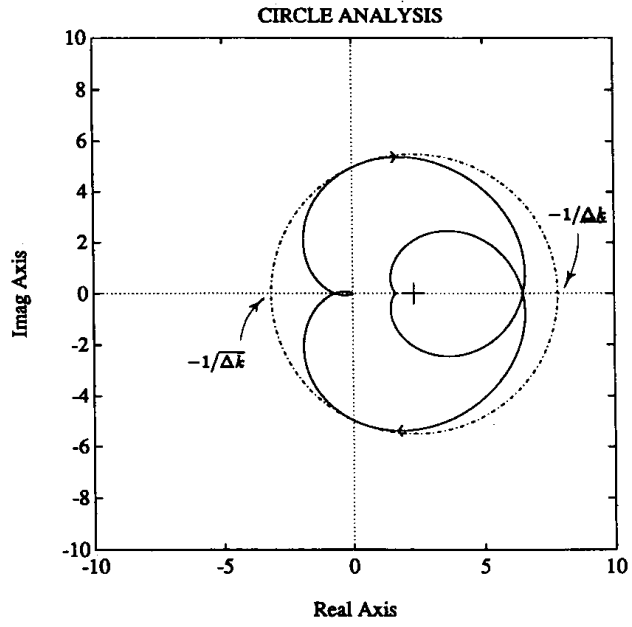

Fig. 4 Circle analysis.

\section{Positive Real Analysis}

Positive real analysis determines stability bounds by drawing straight-lines that lie to the left or right of the Nyquist diagram (without touching it). It is equivalent to the limit of the circle criterion as the center of the circle moves toward infinity along the positive or negative real axis and will always give less conservative bounds. For the Nyquist diagram of $\tilde{G}(s)$, the intersection of the line to the left of the Nyquist plot with the negative real axis equals $-1 \sqrt{\Delta k}$. The intersection of the line to the right of the Nyquist plot with the positive real axis equals $-1 / \Delta k$. This analysis is shown in Figure 5. It follows that $\overline{\Delta k}=0.5277$ and $\Delta k=-0.1522$. Hence, using positive real analysis, stability is guaranteed for

$$
0.4478 \leq k \leq 1.1277 \text {. }
$$

\section{Popov Analysis}

Popov analysis is a test that determines a stability bound from a modified Nyquist diagram, namely the Popov plot, $\omega \operatorname{Im}[\tilde{G}(j \omega)]$ vs. $\operatorname{Re}[\tilde{G}(j \omega)]$ for $\omega \geq 0$. This analysis requires finding lines (Popov lines) that intersect the negative or positive real axis at a point that is to the left of the Popov plot but as close to the origin as possible. The slope of these lines are $-1 / \bar{N}$ and $-1 / \underline{N}$ where $\bar{N}$ and $\underline{N}$ are the Popov multipliers. The Popov test is equivalent to the positive real test if the lines are chosen to be vertical. For the Popov diagram of $\tilde{G}(s)$, the intersection of the line to the left of the Popov plot with the negative real axis equals $-1 / \overline{\Delta k}$. The intersection of the line to the right of the Popov plot with the positive real axis equals $-1 / \underline{\Delta k}$. This analysis is shown in Figure 6. It follows that $\overline{\Delta k}=1.4660$ and $\underline{\Delta k}=0.1541$ and the corresponding Popov multipliers are respectively $\bar{N}=0.7999$ and $N=-0.2755$. Hence, using Popov analysis, stability is guaranteed for

$$
0.4459 \leq k \leq 2.0660 .
$$

Note that these bounds are identical to the exact bounds (2), at least to four-digit precision for the lower bound and five digit precision for the upper bound. Hence, for this example, Popov

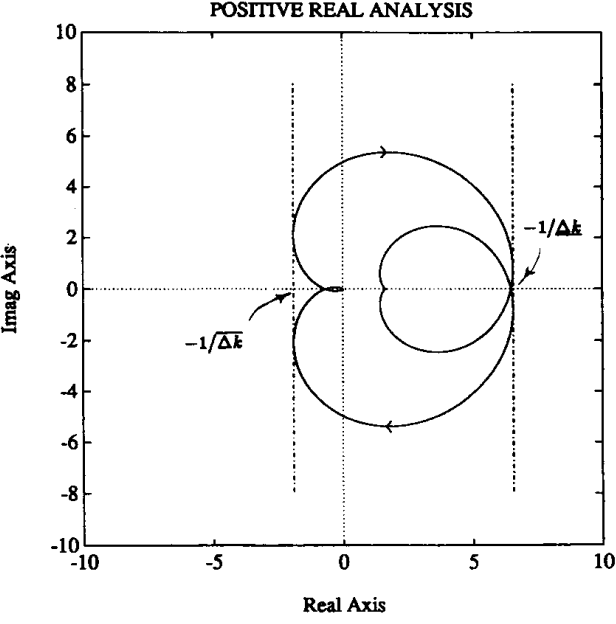

Fig. 5 Positive real analysis.

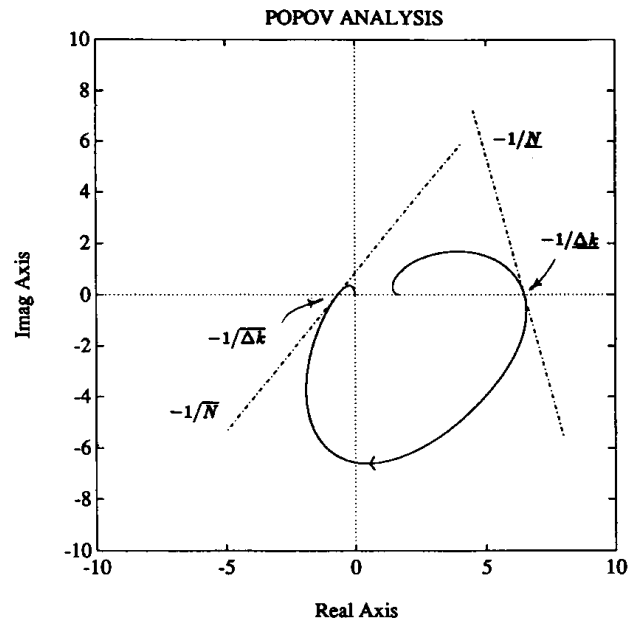

Fig. 6 Popov analysis.

analysis yielded highly nonconservative results. This is not surprising since, as mentioned in the Introduction, the Popov result is based upon a parameter-dependent Lyapunov function which severely restricts the allowable time variation of the uncertain parameters and hence closely approximates real parameter uncertainty within robustness analysis.

\section{Conclusion}

We have shown by means of a simple two-mass/spring example with uncertain stiffness that small gain modeling of constant real parameter uncertainty can be extremely conservative. An alternative approach to the phase information/real parameter uncertainty problem using Popov analysis and related parameter-dependent Lyapunov functions was shown to be significantly less conservative. Although Popov analysis was traditionally developed to analyze stability of a system with a single, memoryless nonlinear element in a feedback configuration, recent results have reinterpreted Popov analysis to handle the problem of robust stability involving constant, linear uncertainty $[3,4]$. 
The results here demonstrate the somewhat overlooked fact that Popov analysis can be very nonconservative when applied to the analysis of linear systems with linear uncertainty.

Finally, it should be acknowledged that the results of $[3,4]$ allow Popov analysis to be used to synthesize robust controllers. This problem of robust control can, of course, be alternatively approached using adaptive control techniques $[9,10]$ which implicitly or explicitly identify the model uncertainty. It is possible that the results discussed in $[3,4]$ can be used as a basis for using Popov analysis to determine the stability and robustness properties of adaptive controllers.

\section{REFERENCES}

[1] K. S. Narendra and J. H. Taylor, Frequency Domain Criteria for Absolute Stability, New York: Academic Press, 1973.

[2] D. S. Bernstein, W. M. Haddad, and D. C. Hyland "Small Gain Versus Positive Real Modeling of Real Parameter Uncertainty," AIAA J. Guid. Contr. Dyn., vol. 15, pp. 538-540, 1992.

[3] W. M. Haddad and D. S. Bernstein, "Explicit Construction of Quadratic Lyapunov Functions for the Small Gain, Positivity, Circle, and PopovTheorems and Their Application to Robust Stability," Proc. IEEE Conf. Dec. Contr., pp. 2618-2623, Brighton, U.K., December 1991. (Also submitted to Int. J. Robust and Nonlinear Control)

[4] W. M. Haddad and D. S. Bernstein, "Parameter-Dependent Lyapunov Functions, Constant Real Parameter Uncertainty, and the Popov Criterion in Robust Analysis and Synthesis: Part I, Part II," Proc. IEEE Conf. Dec. Contr., Brighton, U.K., December 1991, pp. 2274-2279, pp. 2632-2633. (Also to be published inIEEE Trans. Autom. Contr.)

[5] B. Wie and D. S. Bernstein, "A Benchmark Problem for Robust Control Design," Proc. Amer. Contr. Conf., San Diego, CA, May, 1990, pp. 961-962.

[6] E. G. Collins, Jr., J. A. King, and D. S. Bernstein, "Robust Control Design for a Benchmark Problem Using the Maximum Entropy Approach," Proc. Amer. Contr. Conf., Boston, MA, June 1991, pp. 1935-1936.

[7] E. G. Collins, Jr., J. A. King, and D. S. Bernstein, "Application of Maximum Entropy/Optimal Projection Design Synthesis to the Benchmark Problem," AIAA J. Guid. Contr. Dyn., to be published.

[8] J. C. Doyle, "Analysis of Feedback Systems with Structured Uncertainties," IEE Proc., Part D. Vol. 129, 1982, pp. 242-250.

[9] K. J. Astrom and B. Wittenmark, Adaptive Control, New York: Addison Wesley, 1989.

[10] K. S. Narendra and A. M. Annaswamy, Stable Adaptive Systems, Englewood Cliffs, New Jersey: Prentice Hall, 1989. 